\title{
One-loop analysis with nonlocal boundary conditions
}

\author{
Giampiero Esposito* and Elisabetta Di Grezia ${ }^{\dagger}$ \\ Istituto Nazionale di Fisica Nucleare, Sezione di Napoli, Complesso Universitario di Monte S. Angelo, \\ Via Cintia Edificio 6, 80126 Napoli, Italy
}

(Received 10 December 2018; published 13 March 2019)

\begin{abstract}
In the 1980s, Schröder studied a quantum mechanical model where the stationary states of Schrödinger's equation obey nonlocal boundary conditions on a circle in the plane. For such a problem, we perform a detailed one-loop calculation for three choices of the kernel characterizing the nonlocal boundary conditions. In such cases, the $\zeta(0)$ value is found to coincide with the one resulting from Robin boundary conditions. The detailed technique here developed may be useful for studying one-loop properties of quantum field theory and quantum gravity if nonlocal boundary conditions are imposed.
\end{abstract}

DOI: $10.1103 /$ PhysRevD.99.065004

\section{INTRODUCTION}

In the late 1980s, motivated by physical models of Bose condensation and mathematical study of Schrödinger operators, the work in Ref. [1] studied spectral properties of the Laplace operator with nonlocal boundary conditions. Within this framework, on considering the region

$$
B_{R} \equiv\left\{x, y: x^{2}+y^{2} \leq R^{2}\right\},
$$

one builds, out of a function $q$ which is both Lebesgue summable and square-integrable on the real line, the periodic function

$$
q_{R}(x) \equiv \frac{1}{2 \pi R} \sum_{l=-\infty}^{\infty} e^{i l x / R} \int_{-\infty}^{\infty} e^{-i l y / R} q(y) d y
$$

which has period $2 \pi R$ and approaches $q$ if $R \rightarrow \infty$. On using polar coordinates $(r, \varphi)$, the nonlocal boundary-value problem studied in Ref. [1] reads as

$$
\begin{array}{r}
-\left(\frac{\partial^{2}}{\partial r^{2}}+\frac{1}{r} \frac{\partial}{\partial r}+\frac{1}{r^{2}} \frac{\partial^{2}}{\partial \varphi^{2}}\right) u=E u, \quad \forall r<R, \\
\frac{\partial u}{\partial r}+R \int_{-\pi}^{\pi} q_{R}(R(\varphi-\theta)) u(R, \theta) d \theta=0 .
\end{array}
$$

*gesposit@na.infn.it

digrezia@na.infn.it

Published by the American Physical Society under the terms of the Creative Commons Attribution 4.0 International license. Further distribution of this work must maintain attribution to the author(s) and the published article's title, journal citation, and DOI. Funded by SCOAP ${ }^{3}$.
The resulting spectrum has both a positive $(E>0)$ and a negative $(E<0)$ part. For $E>0$, the solutions regular at the origin $r=0$ are factorized in the form

$$
u_{l, E}(r, \varphi)=J_{l}(r \sqrt{E}) e^{i l \varphi}, \quad l \in Z,
$$

the $J_{l}$ being Bessel functions of the first kind. On defining the dimensionless variable $h \equiv R \sqrt{E}$, Eq. (1.4) takes eventually the form [1]

$$
G_{l}(h) \equiv h J_{l}^{\prime}(h)+R \tilde{q}\left(\frac{l}{R}\right) J_{l}(h)=0,
$$

where $\tilde{q}$ is the Fourier transform of $q$, i.e., [1]

$$
\tilde{q}\left(\frac{l}{R}\right)=\int_{-\infty}^{\infty} q(x) e^{-i \frac{l}{R} x} d x
$$

We note from (1.6) that $\tilde{q}$ must have dimension length ${ }^{-1}$, and hence $q$ must have dimension length ${ }^{-2}$.

In the present paper, we have tried to work out the oneloop properties pertaining to the problem defined by Eqs. (1.3) and (1.4). In the physics-oriented literature, one-loop calculations are more frequently performed in the case of quantum field theories, but the quantum mechanical framework is already of interest [2], and may provide valuable information on the behaviour of solutions of elliptic equations under a scale dilation. Such a property is neatly described by the $\zeta(0)$ value, where $\zeta$ is the spectral (or generalized) $\zeta$-function of the elliptic operator $A$ under consideration, defined by

$$
\zeta_{A}(s) \equiv \operatorname{Tr}_{L^{2}}\left(A^{-s}\right)=\sum_{n} \lambda_{n}^{-s},
$$


where each eigenvalue $\lambda_{n}$ is counted with its degeneracy. For this purpose, Sec. II outlines the analytic technique of Ref. [3] and its application to our boundary-value problem (1.3) and (1.4). Section III evaluates the first three sums that contribute to the $\zeta(0)$ value, whereas Sec. IV studies the fourth and last sum contributing to $\zeta(0)$, for three choices of the function $q$ and hence of the dimensionless coefficients $\beta_{l}$ from Eqs. (1.6) and (1.7), i.e.,

$$
\beta_{l} \equiv R \tilde{q}\left(\frac{l}{R}\right) .
$$

Concluding remarks are presented in Sec. V, whereas relevant details are given in the Appendix.

\section{II. $\zeta(0)$ VALUE FROM THE $\zeta$-FUNCTION AT LARGE $h$}

For the Laplacian $A$ on the left-hand side of Eq. (1.3), the associated heat equation has a heat kernel $K(\xi, \eta ; t)$, whose diagonal $K(\xi, \xi ; t)$ yields, upon integration over the whole region $B_{R}$ in (1.1), the integrated heat kernel (for gauge theories, the trace to be integrated is instead the fiber trace of the heat-kernel diagonal),

$$
K(t)=\int_{B_{R}} K(\xi, \xi ; t)=\operatorname{Tr}_{L^{2}} e^{-t A},
$$

which has, as $t \rightarrow 0^{+}$, the asymptotic expansion [3-5]

$$
K(t) \sim \sum_{n=0}^{\infty} B_{n} t^{\frac{n}{2}-1}
$$

In our two-dimensional region $B_{R}$, the method used in Ref. [3] considers the so-called spectral $\zeta$-function at large $h$, i.e., $\left[\lambda_{n}\right.$ and $h$ being dimensionless in (2.3)]

$$
\zeta\left(s, h^{2}\right) \equiv \sum_{n}\left(\lambda_{n}+h^{2}\right)^{-s},
$$

which is related to the integrated heat kernel (2.1) by the identity

$$
\int_{0}^{\infty} t e^{-h^{2} t} K(t) d t=\Gamma(2) \zeta\left(2, h^{2}\right) .
$$

If one now inserts into the left-hand side of (2.4) the asymptotic expansion (2.2), one finds

$$
\Gamma(2) \zeta\left(2, h^{2}\right) \sim \sum_{n=0}^{\infty} B_{n} \Gamma\left(1+\frac{n}{2}\right) h^{-n-2} .
$$

On the other hand, on considering the equation (1.6), which is the equation obeyed by the eigenvalues $E=\frac{h^{2}}{R^{2}}$ by virtue of the boundary conditions, one has also the identity (see [3] and our Appendix)
$\Gamma(2) \zeta\left(2, h^{2}\right)=\sum_{l}^{\infty}\left(-N_{l}\right)\left(\frac{1}{2 h} \frac{d}{d h}\right)^{2} \log \left[(i h)^{-l} G_{l}(i h)\right]$,

where the degeneracy $N_{l}$ equals 2 , as is clear from (1.5) (for each value of $l$, there exist 2 linearly independent eigenfunctions proportional to $\cos (l \varphi)$ and $\sin (l \varphi)$, respectively). In light of (2.5) and (2.6), the desired $\zeta(0)$ is the coefficient of $h^{-4}$ in the asymptotic expansion of the righthand side of Eq. (2.6), because $\zeta(0)=B_{2}$ from the formulae [4]

$$
\begin{aligned}
\zeta(s) & =\frac{1}{\Gamma(s)} \int_{0}^{\infty} t^{s-1} K(t) d t \\
& \sim \frac{1}{\Gamma(s)}\left[\sum_{n=0}^{\infty} B_{n} \int_{0}^{1} t^{\frac{n}{2}+s-2} d t+\int_{1}^{\infty} t^{s-1} K(t) d t\right] .
\end{aligned}
$$

In the course of performing sums over all positive and negative values of $l$, it is helpful to exploit the identity

$$
J_{-l}(z)=(-1)^{l} J_{l}(z)
$$

as well as the even nature of $\beta_{l}$ as a function of $l$ (see Sec. IV). This implies that a real root of $G_{l}$ with positive $l$ is also a real root of $G_{l}$ with negative $l$, because

$$
G_{-l}(z)=(-1)^{l} G_{l}(z)
$$

We can therefore limit ourselves to summing over positive values of $l$, writing that, in (2.6),

$$
\begin{aligned}
\sum_{l}^{\infty} & =2 \sum_{l=1}^{\infty}+\text { contribution of }(l=0) \\
& =2 \sum_{l=0}^{\infty}-\text { contribution of }(l=0) .
\end{aligned}
$$

Following Ref. [3], we use in (2.6) and (2.10) the uniform asymptotic expansion of $J_{l}$ and its first derivative $J_{l}^{\prime}$, which involve the polynomials $u_{k}$ and $v_{k}$ occurring below and in the Appendix. On denoting by $C$ a constant, and defining $\alpha_{l}(i h) \equiv \sqrt{l^{2}+h^{2}}$, we obtain

$$
\begin{aligned}
\log \left[(i h)^{-l} G_{l}(i h)\right] \sim & C-l \log \left(l+\alpha_{l}\right)+\frac{1}{2} \log \left(\alpha_{l}\right)+\alpha_{l} \\
& +\log \left[1+\frac{\left(b_{1}+\beta_{l}\right)}{\alpha_{l}}+\frac{\left(b_{2}+\beta_{l} a_{1}\right)}{\alpha_{l}^{2}}\right. \\
& \left.+\frac{\left(b_{3}+\beta_{l} a_{2}\right)}{\alpha_{l}^{3}}+\mathrm{O}\left(\alpha_{l}^{-4}\right)\right],
\end{aligned}
$$

where, having defined the variable 


$$
\tau \equiv \frac{l}{\alpha_{l}}=\frac{l}{\sqrt{l^{2}+h^{2}}},
$$

and exploiting the following among the many Debye-Olver polynomials [6]:

$u_{0}(\tau)=1, \quad u_{1}(\tau)=\frac{\tau}{8}-\frac{5}{24} \tau^{3}$

$u_{2}(\tau)=\frac{9}{128} \tau^{2}-\frac{77}{192} \tau^{4}+\frac{385}{1152} \tau^{6}$,

$v_{0}(\tau)=1, \quad v_{1}(\tau)=-\frac{3}{8} \tau+\frac{7}{24} \tau^{3}$,

$v_{2}(\tau)=-\frac{15}{128} \tau^{2}+\frac{99}{192} \tau^{4}-\frac{455}{1152} \tau^{6}$

$v_{3}(\tau)=-\frac{105}{1024} \tau^{3}+\frac{5577}{5120} \tau^{5}-\frac{6545}{3072} \tau^{7}+\frac{95095}{82944} \tau^{9}$

the polynomials $a_{1}, a_{2}, b_{1}, b_{2}, b_{3}$ are evaluated according to the definition

$$
a_{k}(\tau)=\frac{u_{k}(\tau)}{\tau^{k}}, \quad b_{k}(\tau)=\frac{v_{k}(\tau)}{\tau^{k}}
$$

which implies the simple but very helpful relations

$$
\frac{u_{k}}{l^{k}}=\frac{a_{k}}{\left(\alpha_{l}\right)^{k}}, \quad \frac{v_{k}}{l^{k}}=\frac{b_{k}}{\left(\alpha_{l}\right)^{k}} .
$$

\section{CONTRIBUTIONS INDEPENDENT OF $\beta_{l}$}

By virtue of (2.6) and (2.11) the three contributions independent of $\beta_{l}$ are obtained by applying twice the operator $\frac{1}{2 h} \frac{d}{d h}$ to the first line on the right-hand side of (2.11). For this purpose, we need the following identities:

$$
\begin{aligned}
\frac{1}{2 h} \frac{d \alpha_{l}}{d h} & =\frac{1}{2 \alpha_{l}} \\
\left(\frac{1}{2 h} \frac{d}{d h}\right)^{2} \log \left(l+\alpha_{l}\right) & =-\frac{1}{4}\left(l+\alpha_{l}\right)^{-2}\left[2 \alpha_{l}^{-2}+l \alpha_{l}^{-3}\right] \\
\left(\frac{1}{2 h} \frac{d}{d h}\right)^{2} \log \left(\alpha_{l}\right) & =-\frac{1}{2} \alpha_{l}^{-4} \\
\left(\frac{1}{2 h} \frac{d}{d h}\right)^{2} \alpha_{l} & =-\frac{1}{4} \alpha_{l}^{-3}
\end{aligned}
$$

Thus, upon applying the split (2.10), the terms independent of $\beta_{l}$ are obtained by taking twice [from the factor 2 multiplying $\sum_{l=0}^{\infty}$ in (2.10)] the following sums:

$$
\begin{aligned}
\sigma_{1} & =2 \sum_{l=0}^{\infty}\left(\frac{1}{2 h} \frac{d}{d h}\right)^{2} l \log \left(l+\alpha_{l}\right) \\
& =-\frac{1}{2} \sum_{l=0}^{\infty} l\left(l+\alpha_{l}\right)^{-2}\left[2 \alpha_{l}^{-2}+l \alpha_{l}^{-3}\right], \\
\sigma_{2} & =-\sum_{l=0}^{\infty}\left(\frac{1}{2 h} \frac{d}{d h}\right)^{2} \log \left(\alpha_{l}\right)=\frac{1}{2} \sum_{l=0}^{\infty} \alpha_{l}^{-4}, \\
\sigma_{3} & =-2 \sum_{l=0}^{\infty}\left(\frac{1}{2 h} \frac{d}{d h}\right)^{2} \alpha_{l}=\frac{1}{2} \sum_{l=0}^{\infty} \alpha_{l}^{-3} .
\end{aligned}
$$

\section{A. Contribution of $\sigma_{1}$}

The sums (3.5)-(3.7) can be studied in a thorough way with the help of the Euler-Maclaurin summation formula [7]. This states that, if $f$ is a real- or complex-valued function defined on $[0, \infty)$, and if its derivatives of even order are absolutely integrable on $(0, \infty)$, one has, for $n=1,2, \ldots$,

$$
\begin{array}{rl}
\sum_{l=0}^{n} & f(l)-\int_{0}^{n} f(y) d y \\
= & \frac{1}{2}[f(0)+f(n)] \\
& +\sum_{s=1}^{m-1} \frac{\tilde{B}_{2 s}}{(2 s) !}\left[f^{(2 s-1)}(n)-f^{(2 s-1)}(0)\right]+R_{m}(n),
\end{array}
$$

where the Bernoulli numbers $\tilde{B}_{s}$ are defined by the expansion

$$
\frac{t}{\left(e^{t}-1\right)}=\sum_{s=0}^{\infty} \tilde{B}_{s} \frac{t^{s}}{s !}, \quad|t|<2 \pi,
$$

while the remainder $R_{m}(n)$ is majorized according to [7]

$$
\left|R_{m}(n)\right| \leq\left(2-2^{1-m}\right) \frac{\left|\tilde{B}_{2 m}\right|}{(2 m) !} \int_{0}^{n}\left|f^{(2 m)}(y)\right| d y .
$$

As $n$ approaches $\infty$, Eq. (3.8) provides a very useful asymptotic expansion for the desired sum of the series, i.e.,

$$
\begin{aligned}
\sum_{l=0}^{\infty} f(l) \sim & \int_{0}^{\infty} f(y) d y+\frac{1}{2} f(0) \\
& +\sum_{s} \frac{\tilde{B}_{2 s}}{(2 s) !}\left[f^{(2 s-1)}(\infty)-f^{(2 s-1)}(0)\right] .
\end{aligned}
$$

The integral on the right-hand side of (3.11) can be evaluated or studied in a qualitative way, while the derivatives of odd order at 0 and at $\infty$ can be obtained in a systematic way. We refer the reader to the last chapter 
of the book by Hardy [8] for a thorough analysis of the Euler-Maclaurin formula. form

For our purposes, after having re-expressed $\sigma_{1}$ in the

$$
\sigma_{1}=\sum_{l=0}^{\infty}[F(l ; h)+H(l ; h)]
$$

where we have set

$$
\begin{aligned}
& F(l ; h) \equiv-l\left(l^{2}+h^{2}\right)^{-1}\left(l+\sqrt{l^{2}+h^{2}}\right)^{-2}, \\
& H(l ; h) \equiv-\frac{l^{2}}{2}\left(l^{2}+h^{2}\right)^{-\frac{3}{2}}\left(l+\sqrt{l^{2}+h^{2}}\right)^{-2},
\end{aligned}
$$

we now take the limit as $n \rightarrow \infty$ in Eq. (3.8), with $f(l)$ replaced by $F(l ; h)+H(l ; h)$. We then find that, in (3.11), only the first derivative of $F$ at $l=0$ gives a contribution proportional to $h^{-4}$, and indeed equal to

$$
\delta_{1}=\frac{1}{2 !} \tilde{B}_{2}\left(-F^{\prime}(0 ; h)\right)=\frac{1}{12} h^{-4} .
$$

\section{B. Contributions of $\sigma_{2}$ and $\sigma_{3}$}

We now rely again upon the limit as $n \rightarrow \infty$ of Eq. (3.8). By virtue of (3.11), only half the value at $l=0$ of $\frac{1}{2} \alpha_{l}^{-4}$ contributes to the $h^{-4}$ term in $\sigma_{2}$, i.e.,

$$
\delta_{2}=\frac{1}{2} \frac{1}{2} h^{-4}=\frac{1}{4} h^{-4},
$$

whereas $\sigma_{3}$ gives a vanishing contribution to the term proprtional to $h^{-4}$

$$
\delta_{3}=0
$$

\section{CONTRIBUTION FROM THE $\beta_{l}$ COEFFICIENTS}

We now aim at studying the contribution of the second and third line of the asymptotic expansion (2.11) to Eq. (2.6). For this purpose, on the one hand we denote by $\Omega$ all terms added to 1 within the square brackets in (2.11), and consider the asymptotic expansion

$$
\begin{aligned}
\log (1+\Omega) & \sim \Omega-\frac{\Omega^{2}}{2}+\frac{\Omega^{3}}{3}+\mathrm{O}\left(\Omega^{4}\right) \\
& \sim \frac{\kappa_{1}}{\alpha_{l}}+\frac{\kappa_{2}}{\left(\alpha_{l}\right)^{2}}+\frac{\kappa_{3}}{\left(\alpha_{l}\right)^{3}}+\mathrm{O}\left(\alpha_{l}^{-4}\right),
\end{aligned}
$$

where $\kappa_{1} \equiv b_{1}+\beta_{l}$,

$\kappa_{2} \equiv\left(b_{2}+\beta_{l} a_{1}\right)-\frac{1}{2}\left(b_{1}+\beta_{l}\right)^{2}$,

$\kappa_{3} \equiv b_{3}+\beta_{l} a_{2}-\left(b_{1}+\beta_{l}\right)\left(b_{2}+\beta_{l} a_{1}\right)+\frac{1}{3}\left(b_{1}+\beta_{l}\right)^{3}$.

On the other hand, it is clear that no further progress can be made without explicit forms of the $\beta_{l}$ coefficients. For example, we find from (1.7) and (1.9)

$$
\begin{aligned}
& q(x)=\frac{1}{R^{2}} e^{-\frac{x^{2}}{R^{2}}} \Rightarrow \beta_{l}=\frac{1}{\sqrt{2}} e^{-\frac{l^{2}}{4}}, \\
& q(x)=\sqrt{\frac{\pi}{2}} \frac{1}{R^{2}} e^{-\frac{|x|}{R}} \Rightarrow \beta_{l}=\frac{1}{\left(1+l^{2}\right)}, \\
& q(x)=\sqrt{\frac{2}{\pi}} \frac{1}{x^{2}} \Rightarrow \beta_{l}=-l \operatorname{sgn}(l), \\
& q(x)=\sqrt{\frac{2}{\pi}} \frac{1}{\left(R^{2}+x^{2}\right)} \Rightarrow \beta_{l}=e^{-|l|} .
\end{aligned}
$$

Here we consider first the choice of $\beta_{l}$ in Eq. (4.6), and exploit the formulae (2.6), (2.11)-(3.1), and (4.1)-(4.4), arriving therefore at the sums (see details below)

$$
\begin{aligned}
\left(\hat{\sigma}_{4}\right)_{I} & \equiv-2 \sum_{l=0}^{\infty}\left(\frac{1}{2 h} \frac{d}{d h}\right)^{2} \frac{\kappa_{1}}{\alpha_{l}} \\
& =\frac{9}{16} \sum_{l=0}^{\infty} \alpha_{l}^{-5}-\frac{35}{16} \sum_{l=0}^{\infty} l^{2} \alpha_{l}^{-7}-\frac{3}{2} \sum_{l=0}^{\infty} \frac{\alpha_{l}^{-5}}{\left(l^{2}+1\right)} \\
\left(\hat{\sigma}_{4}\right)_{I I} & \equiv-2 \sum_{l=0}^{\infty}\left(\frac{1}{2 h} \frac{d}{d h}\right)^{2} \frac{\kappa_{2}}{\left(\alpha_{l}\right)^{2}}
\end{aligned}
$$

supplemented, in principle, by infinitely many other terms, i.e.,

$$
\left(\hat{\sigma}_{4}\right)_{m} \equiv-2 \sum_{l=0}^{\infty}\left(\frac{1}{2 h} \frac{d}{d h}\right)^{2} \frac{\kappa_{m}}{\left(\alpha_{l}\right)^{m}}, \quad \forall m=3,4, \ldots, \infty
$$

In the formula (4.10), it is helpful to use (4.3) where we reexpress $a_{1}, b_{1}$, and $b_{2}$ in the form

$$
\begin{aligned}
a_{1} & =\sum_{r=0}^{1} a_{1 r}\left(\frac{l}{\alpha_{l}}\right)^{2 r}, \quad b_{1}=\sum_{r=0}^{1} b_{1 r}\left(\frac{l}{\alpha_{l}}\right)^{2 r}, \\
b_{2} & =\sum_{r=0}^{2} b_{2 r}\left(\frac{l}{\alpha_{l}}\right)^{2 r},
\end{aligned}
$$


where the numerical coefficients $a_{1 r}, b_{1 r}$ and $b_{2 r}$ can be read off from (2.13), (2.15), and (2.16). Thus, a patient calculation shows that (the superscript $(l)$ denotes here dependence on $l$ )

$$
\frac{\kappa_{2}}{\left(\alpha_{l}\right)^{2}}=\sum_{r=0}^{2} \kappa_{2 r}^{(l)} l^{2 r} \alpha_{l}^{-2 r-2}
$$

where

$$
\begin{aligned}
\kappa_{20}^{(l)} & =b_{20}+\beta_{l}\left(a_{10}-b_{10}\right)-\frac{1}{2}\left(\left(b_{10}\right)^{2}+\left(\beta_{l}\right)^{2}\right) \\
& =-\frac{3}{16}+\frac{1}{2} \beta_{l}\left(1-\beta_{l}\right), \\
\kappa_{21}^{(l)} & =b_{21}+\beta_{l}\left(a_{11}-b_{11}\right)-b_{10} b_{11}=\frac{5}{8}-\frac{1}{2} \beta_{l}, \\
\kappa_{22}^{(l)} & =b_{22}-\frac{1}{2}\left(b_{11}\right)^{2}=-\frac{7}{16},
\end{aligned}
$$

and, hence, by repeated application of (3.1), we obtain from (4.10) and (4.13)

$$
\begin{aligned}
\left(\hat{\sigma}_{4}\right)_{I I}= & -2 \sum_{l=0}^{\infty} \sum_{r=0}^{2}(r+1)(r+2) \kappa_{2 r}^{(l)} l^{2 r} \alpha_{l}^{-2 r-6} \\
= & \frac{3}{4} \sum_{l=0}^{\infty} \alpha_{l}^{-6}-\frac{15}{2} \sum_{l=0}^{\infty} l^{2} \alpha_{l}^{-8}+\frac{21}{2} \sum_{l=0}^{\infty} l^{4} \alpha_{l}^{-10} \\
& -2 \sum_{l=0}^{\infty} \beta_{l}\left(1-\beta_{l}\right) \alpha_{l}^{-6}+6 \sum_{l=0}^{\infty} \beta_{l} l^{2} \alpha_{l}^{-8}
\end{aligned}
$$

\section{A. Effect of Eq. (4.9)}

In Eq. (4.9), by virtue of the remarkable formula [3],

$\sum_{l=0}^{\infty} l^{2 k} \alpha_{l}^{-2 k-m}=\frac{\Gamma\left(k+\frac{1}{2}\right) \Gamma\left(\frac{m}{2}-\frac{1}{2}\right)}{2 \Gamma\left(k+\frac{m}{2}\right)} x^{1-m}, \quad k=1,2,3, \ldots$,

$\Gamma$ being the standard notation for the $\Gamma$-function, we find

$$
\sum_{l=0}^{\infty} l^{2} \alpha_{l}^{-7}=\frac{2}{15} h^{-4}
$$

a result which agrees with the application of Eq. (3.11). Moreover, the asymptotic expansion (3.11) implies that the first sum on the second line of right-hand side of (4.9) is equal to

$$
\sum_{l=0}^{\infty} \alpha_{l}^{-5} \sim \int_{0}^{\infty}\left(y^{2}+h^{2}\right)^{-\frac{5}{2}} d y+\frac{1}{2} h^{-5},
$$

where, on defining $Y \equiv \frac{y}{h}$, we find

$$
\int_{0}^{\infty}\left(y^{2}+h^{2}\right)^{-\frac{5}{2}} d y=h^{-4} \int_{0}^{\infty}\left(Y^{2}+1\right)^{-\frac{5}{2}} d Y=\frac{2}{3} h^{-4} .
$$

It is clear once more that $h$ plays the role of regularizing parameter, since without it the integral (4.21), and many of the integrals below, would not exist. Last, the third sum on the right-hand side of (4.9) is again studied with the help of (3.11), and we find

$$
\sum_{l=0}^{\infty} \frac{\alpha_{l}^{-5}}{\left(l^{2}+1\right)} \sim W(h)+\frac{1}{2} h^{-5},
$$

where

$$
\begin{aligned}
W(h) & \equiv \int_{0}^{\infty} \frac{\left(y^{2}+h^{2}\right)^{-\frac{5}{2}}}{\left(y^{2}+1\right)} d y=h^{-4} \int_{0}^{\infty} \frac{\left(Y^{2}+1\right)^{-\frac{5}{2}}}{\left(h^{2} Y^{2}+1\right)} d Y \\
& =h^{-4} \frac{\left[\left(2-5 h^{2}\right) \sqrt{h^{2}-1}+3 h^{4} \arccos \frac{1}{h}\right]}{3\left(h^{2}-1\right)^{\frac{5}{2}}},
\end{aligned}
$$

and hence no contribution to $h^{-4}$ arises from (4.22) at large $h$.

\section{B. Contribution of (4.17) and $\zeta(0)$ value}

In Eq. (4.17), by virtue of (4.18), the sums

$$
\sum_{l=0}^{\infty} l^{2} \alpha_{l}^{-8}, \quad \sum_{l=0}^{\infty} l^{4} \alpha_{l}^{-10}
$$

do not contribute to $h^{-4}$, while Eq. (3.11) tells us that

$$
\begin{aligned}
\sum_{l=0}^{\infty} \alpha_{l}^{-6} & \sim \int_{0}^{\infty}\left(y^{2}+h^{2}\right)^{-3} d y+\frac{1}{2} h^{-6} \\
& =h^{-5} \int_{0}^{\infty}\left(Y^{2}+1\right)^{-3} d Y+\frac{1}{2} h^{-6},
\end{aligned}
$$

and hence (4.24) does not contribute to $h^{-4}$ either. Furthermore, the last two sums on the right-hand side of (4.17), which contain the effect of $\beta_{l}$, with the particular choice (4.6) for this coefficient are found to involve

$$
\begin{aligned}
\Sigma_{\beta}^{1} & \equiv \sum_{l=0}^{\infty} \frac{l^{2}}{\left(l^{2}+1\right)^{2}} \alpha_{l}^{-6} \sim \int_{0}^{\infty} \frac{y^{2}}{\left(y^{2}+1\right)^{2}}\left(y^{2}+h^{2}\right)^{-3} d y \\
& =h^{-3} \int_{0}^{\infty} \frac{Y^{2}\left(Y^{2}+1\right)^{-3}}{\left(h^{2} Y^{2}+1\right)^{2}} d Y=h^{-3} \frac{(1+4 h) \pi}{16(h+1)^{4}}
\end{aligned}
$$




$$
\begin{aligned}
\Sigma_{\beta}^{2} & \equiv \sum_{l=0}^{\infty} \frac{l^{2}}{\left(l^{2}+1\right)} \alpha_{l}^{-8} \sim \int_{0}^{\infty} \frac{y^{2}}{\left(y^{2}+1\right)}\left(y^{2}+h^{2}\right)^{-4} d y \\
& =h^{-5} \int_{0}^{\infty} \frac{Y^{2}\left(Y^{2}+1\right)^{-4}}{\left(h^{2} Y^{2}+1\right)} d Y=h^{-5} \frac{[1+h(4+5 h)] \pi}{32(h+1)^{4}},
\end{aligned}
$$

none of which contains terms proportional to $h^{-4}$ at large $\mathrm{h}$.

The contributions to $h^{-4}$ arising from all terms in (4.11) are found to vanish with the same procedure just adopted in studying all terms in Eq. (4.17), and hence we find from the second and third line of (2.11) a contribution to $h^{-4}$ equal to

$$
\delta_{4}=\left(\frac{9}{16} \frac{2}{3}-\frac{35}{16} \frac{2}{15}\right) h^{-4}=\frac{1}{12} h^{-4} .
$$

Eventually, we obtain from Eqs. (2.10), (3.15)-(3.17) and (4.27)

$$
\zeta(0)=2\left(\frac{1}{12}+\frac{1}{4}+\frac{1}{12}\right)-\frac{1}{2}=\frac{5}{6}-\frac{1}{2}=\frac{1}{3},
$$

where $-\frac{1}{2}$ is the term denoted in $(2.10)$ by minus the contribution of $(l=0)$, and arises from $\sigma_{2}$ in (3.6).

\section{Other choices of $\boldsymbol{\beta}_{l}$}

For a generic choice of $\beta_{l}$ coefficient, our Eq. (4.9) gets replaced by

$$
\left(\hat{\sigma}_{4}\right)_{I}=\frac{9}{16} \sum_{l=0}^{\infty} \alpha_{l}^{-5}-\frac{35}{16} \sum_{l=0}^{\infty} l^{2} \alpha_{l}^{-7}-\frac{3}{2} \sum_{l=0}^{\infty} \beta_{l} \alpha_{l}^{-5} .
$$

If $\beta_{l}$ is taken in the form (4.5), we find, by virtue of (3.11), the asymptotic expansion $\left(K_{n}\right.$ being the standard notation for modified Bessel functions of second kind and order $n$ )

$$
\begin{aligned}
\sum_{l=0}^{\infty} \beta_{l} \alpha_{l}^{-5} \sim & \frac{1}{\sqrt{2}} \int_{0}^{\infty} \frac{e^{-\frac{y^{2}}{4}}}{\left(y^{2}+h^{2}\right)^{\frac{5}{2}}} d y+\frac{1}{2 \sqrt{2}} h^{-5} \\
= & \frac{1}{\sqrt{2}} \frac{1}{48 h^{2}} e^{\frac{h^{2}}{8}}\left[h^{2} K_{0}\left(\frac{h^{2}}{8}\right)-\left(h^{2}-4\right) K_{1}\left(\frac{h^{2}}{8}\right)\right] \\
& +\frac{1}{2 \sqrt{2}} h^{-5},
\end{aligned}
$$

which has no term proportional to $h^{-4}$, while $\beta_{l}$ in the form (4.8) leads to

$$
\sum_{l=0}^{\infty} \beta_{l} \alpha_{l}^{-5} \sim h^{-4} \int_{0}^{\infty} \frac{e^{-h Y}}{\left(Y^{2}+1\right)^{\frac{5}{2}}} d Y+\frac{1}{2}\left(1+\tilde{B}_{2}\right) h^{-5},
$$

and the integral on the right-hand side of (4.31) does not have a term proportional to $h^{-4}$ at large $h$, either. Moreover, the last two sums in (4.17) suggest introducing the notation

$$
\begin{aligned}
& A\left(\beta_{l}\right) \equiv \sum_{l=0}^{\infty} \beta_{l}\left(\beta_{l}-1\right) \alpha_{l}^{-6}, \\
& B\left(\beta_{l}\right) \equiv \sum_{l=0}^{\infty} \beta_{l} l^{2} \alpha_{l}^{-8} .
\end{aligned}
$$

Now we find, for the two choices of $\beta_{l}$ according to (4.5) or (4.8),

$$
\begin{aligned}
A\left(\frac{1}{\sqrt{2}} e^{-\frac{l^{2}}{4}}\right)= & \frac{1}{2} \sum_{l=0}^{\infty} e^{-\frac{l^{2}}{2}} \alpha_{l}^{-6}-\frac{1}{\sqrt{2}} \sum_{l=0}^{\infty} e^{-\frac{l^{2}}{4}} \alpha_{l}^{-6} \\
\sim & \frac{1}{2}\left[h^{-5} \int_{0}^{\infty} \frac{e^{-\frac{h^{2} Y^{2}}{2}}}{\left(Y^{2}+1\right)^{3}} d Y+\frac{1}{2} h^{-6}\right] \\
& -\frac{1}{\sqrt{2}}\left[h^{-5} \int_{0}^{\infty} \frac{e^{-\frac{h^{2} Y^{2}}{4}}}{\left(Y^{2}+1\right)^{3}} d Y+\frac{1}{2} h^{-6}\right], \\
A\left(e^{-l}\right)= & \sum_{l=0}^{\infty} e^{-2 l} \alpha_{l}^{-6}-\sum_{l=0}^{\infty} e^{-l} \alpha_{l}^{-6} \\
\sim & h^{-5}\left[\int_{0}^{\infty} \frac{\left(e^{-2 h Y}-e^{-h Y}\right)}{\left(Y^{2}+1\right)^{3}} d Y\right]+\frac{\tilde{B}_{2}}{2} h^{-6},
\end{aligned}
$$

$$
\begin{aligned}
& B\left(\frac{1}{\sqrt{2}} e^{-\frac{l^{2}}{4}}\right)=\frac{1}{\sqrt{2}} \sum_{l=0}^{\infty} e^{-\frac{l^{2}}{4}} l^{2} \alpha_{l}^{-8} \\
& \sim \frac{h^{-5}}{\sqrt{2}} \int_{0}^{\infty} e^{-\frac{h^{2} Y^{2}}{4}} \frac{Y^{2}}{\left(Y^{2}+1\right)^{4}} d Y, \\
& B\left(e^{-l}\right)=\sum_{l=0}^{\infty} e^{-l} l^{2} \alpha_{l}^{-8} \sim h^{-5} \int_{0}^{\infty} e^{-h Y} \frac{Y^{2}}{\left(Y^{2}+1\right)^{4}} d Y .
\end{aligned}
$$

Among the integrals occurring in (4.34)-(4.37), only those on the right-hand side of (4.34) might give rise to a contribution proportional to $h^{-4}$, because

$$
\begin{aligned}
\int_{0}^{\infty} & \frac{e^{-\frac{y^{2}}{4}}}{\left(y^{2}+h^{2}\right)^{3}} d y \\
= & \frac{1}{64 h^{4}}\left[2\left(6-h^{2}\right) \sqrt{\pi}+\frac{1}{h}\left(12-4 h^{2}+h^{4}\right) e^{\frac{h^{2}}{4}} \pi\right. \\
& \left.-\left(12-4 h^{2}+h^{4}\right) e^{\frac{h^{2}}{4}} \pi \operatorname{Erf}\left(\frac{h}{2}\right)\right]
\end{aligned}
$$

However, the constant coefficients within square brackets in (4.38) add up to zero, so that no term proportional to $h^{-4}$ actually occurs at large $h$. Such a kind of integral may be studied with the help of complex integration, because

$$
\int_{0}^{\infty} \frac{e^{-\frac{y^{2}}{4}}}{\left(y^{2}+h^{2}\right)^{3}} d y=\frac{1}{64} \int_{-\infty}^{\infty} \frac{e^{-Y^{2}}}{\left(Y^{2}+\frac{h^{2}}{4}\right)^{3}} d Y .
$$


This suggests considering the following integral:

$$
\int_{\gamma} \varphi(z) d z=\int_{\gamma} \frac{e^{-z^{2}}}{\left(z^{2}+\frac{h^{2}}{4}\right)^{3}} d z
$$

where $z=\rho e^{i \theta}$, and $\gamma$ is a rectangle with a side $\gamma_{1}$ given by the closed interval $[-\rho, \rho]$ on the real line, while the other three sides have equation ( $\varepsilon$ being a positive parameter approaching 0 , and we take $h$ positive as well)

$$
\begin{aligned}
& \gamma_{2}: z=\rho+i \eta, \quad \eta \in\left[0, \frac{h}{2}+\varepsilon\right], \\
& \gamma_{3}: z=x+i\left(\frac{h}{2}+\varepsilon\right), \quad x \in[\rho,-\rho], \\
& \gamma_{4}: z=-\rho+i \eta, \quad \eta \in\left[\frac{h}{2}+\varepsilon, 0\right] .
\end{aligned}
$$

The resulting integrand $\varphi(z)$ has a third-order pole at $z=i \frac{h}{2}$, with residue

$$
\begin{aligned}
\left.\operatorname{Res} \varphi(z)\right|_{z=i \frac{h}{2}} & =\left.\frac{d^{2}}{d z^{2}}\left[\frac{\left(z-i \frac{h}{2}\right)^{3} e^{-z^{2}}}{\left(z-i \frac{h}{2}\right)^{3}\left(z+i \frac{h}{2}\right)^{3}}\right]\right|_{z=i \frac{h}{2}} \\
& =-\frac{i}{h^{5}}\left(h^{4}-4 h^{2}+12\right) e^{\frac{h^{2}}{4}}
\end{aligned}
$$

which is one of the three terms occurring in (4.38).

\section{CONCLUDING REMARKS}

As far as we know, our paper has performed the first $\zeta(0)$ calculation with nonlocal boundary conditions in quantum mechanics. We have proved explicitly that at least three choices of kernel in the nonlocal boundary operator exist [see (4.5), (4.6), and (4.8)] for which the $\zeta(0)$ value coincides with the value resulting from local boundary conditions of the Robin type. Our $\zeta(0)$ value does not describe a one-loop conformal anomaly, as it would be the case in quantum field theory, but it remains relevant for the understanding of scaling properties of the quantum Hamiltonian operator.

It remains to be seen whether, for yet other choices of $\beta_{l}$ in (4.17) and (4.29), a contribution to $\zeta(0)$ can be found which is compatible with (1.7), (1.9), the assumption $q \in L_{1}(\mathbf{R}) \cap L_{2}(\mathbf{R})$ and condition (A7) of the Appendix. This means having to study the sums

$$
\sum_{l=0}^{\infty} \beta_{l} \alpha_{l}^{-5}, \quad \sum_{l=0}^{\infty} \beta_{l}\left(\beta_{l}-1\right) \alpha_{l}^{-6}, \quad \sum_{l=0}^{\infty} \beta_{l} l^{2} \alpha_{l}^{-8} .
$$

The mere recourse to the formula [3]

$$
\sum_{l=0}^{\infty} l \alpha_{l}^{-1-n} \sim \frac{h^{1-n}}{\sqrt{\pi}} \sum_{r=0}^{\infty} \frac{2^{r}}{r !} \tilde{B}_{r} x^{-r} \frac{\Gamma\left(\frac{(r+1)}{2}\right) \Gamma\left(\frac{(n-1+r)}{2}\right)}{2 \Gamma\left(\frac{(n+1)}{2}\right)} \cos \left(\frac{r \pi}{2}\right)
$$

suggests a negative answer because, for example, upon requiring

$$
\beta_{l}\left(\beta_{l}-1\right)=\kappa l, \quad \kappa>0,
$$

one finds the positive root

$$
\beta_{l}=\frac{1+\sqrt{1+4 \kappa l}}{2},
$$

which has a growth rate incompatible with (1.7) and (1.9), if one looks for functions $q \in L_{1}(\mathbf{R}) \cap L_{2}(\mathbf{R})$. However, the general starting point should be the asymptotic expansion inspired by (3.11), i.e.,

$$
\begin{aligned}
\sum_{l=0}^{\infty} f(l ; h) \sim & \int_{0}^{\infty} f(y ; h) d y+\frac{1}{2} f(0 ; h) \\
& +\sum_{s} \frac{\tilde{B}_{2 s}}{(2 s) !}\left[f^{2 s-1}(\infty ; h)-f^{2 s-1}(0 ; h)\right],
\end{aligned}
$$

and the application of (5.3) to (5.1) deserves further work.

Furthermore, with the help of the experience gained from our calculation, it should be possible in the near future to investigate the one-loop properties of Euclidean quantum gravity with nonlocal boundary conditions, along the lines of Refs. $[9,10]$, where it was suggested that the Universe might become classical, after a quantum origin, by virtue of a wave function that decays as it occurs in the case of quantum mechanical surface states [1] with nonlocal boundary conditions. In order to help the general reader and stress the relevance of our work, we find it also appropriate to write what follows.

The use of spectral $\zeta$-functions has led to several important developments over the last decades, with application to partition functions of strings and membranes, Casimir effect, relation between the generalized PauliVillars and covariant regularizations, spontaneous compactification in two-dimensional quantum gravity, stability of the rigid membrane, topological symmetry breaking in self-interacting theories, functional determinants for bosonic and fermionic fields, ground-state energy under the influence of external fields, Bose-Einstein condensation of ideal Bose gases under external conditions [11-13]. Furthermore, the work in Ref. [14] obtained heat-kernel coefficients of the Laplace operator on the $D$-dimensional ball, Ref. [15] evaluated Casimir energies for massive fields in the bag, while the Casimir energy for a massive fermionic quantum field with a spherical boundary was obtained in Ref. [16]. The regularization used in our paper, 
which relies as we said on the pioneering work in Ref. [3], was applied successfully in Ref. [17] to the first correct calculation of one-loop conformal anomaly for a massless fermionic field with local boundary conditions, at a time when the powerful geometric formulas in Ref. [4] were not in final form. It is, therefore, encouraging that, after almost thirty years, such a regularization technique is still useful in opening yet new perspectives. For example, it would be interesting to apply it to the Casimir effect [13] in cosmological backgrounds, and to establish a correspondence with yet other models in Ref. [13]. For the gravitational field, one cannot generalize the Schröder scheme by simply studying the eigenvalue problem for an operator of Laplace type on metric perturbations $h_{\mu \nu}$, subject to nonlocal boundary conditions. The reason is that boundary conditions invariant under infinitesimal diffeomorphisms on $h_{\mu \nu}$ take, in field-theoretic language, the form [18]

$$
\begin{gathered}
{\left[(\Pi h)_{i j}\right]_{\partial M}=0,} \\
{\left[\Phi_{\mu}(h)\right]_{\partial M}=0,}
\end{gathered}
$$

where $\Pi$ is a projection operator that picks out only spatial components of $h_{\mu \nu}$, while $\Phi_{\mu}(h)$ is the gauge-fixing functional. Thus, nonlocality in the boundary conditions can only result from the gauge-fixing term; but then the invertible operator on metric perturbations is no longer differential but it belongs to the broader class of pseudodifferential operators $[9,10]$, for which a $\zeta(0)$ calculation is still a challenging task. Thus, new exciting goals are in sight in the area of physical applications of spectral $\zeta$-functions, bearing also in mind the enlightening assessment in Ref. [19].

\section{ACKNOWLEDGMENTS}

The authors are grateful to Dipartimento di Fisica "Ettore Pancini” for hospitality and support.

\section{APPENDIX: CONCEPTS AND FORMULAS FROM COMPLEX ANALYSIS}

We here summarize some concepts and results of complex analysis that are applied in our paper.

If $F$ is an entire function with a countable infinity of zeros $\left\{\mu_{i}\right\}$, the genus of the canonical product for $F$ [20] is the minimum integer $n$ such that

$$
\sum_{i=1}^{\infty} \frac{1}{\left|\mu_{i}\right|^{n+1}}
$$

converges. In particular, if the genus is equal to 1 , this ensures that one can write, for some constant $\gamma$,

$$
F(z)=\gamma z^{m} e^{g(z)} \prod_{i=1}^{\infty}\left(1-\frac{z}{\mu_{i}}\right) e^{\frac{z}{\mu_{i}}},
$$

where the function $g$ is entire. In particular, the function (1.6) occurring in the nonlocal boundary condition is an entire function of genus 1 . Such a property can be checked by pointing out that the zeros of $G_{l}(h)$ at large $h$ are given approximately by

$$
h \sim l \frac{\pi}{2}+\frac{\pi}{4}+\kappa \pi, \quad \kappa \in Z,
$$

because for fixed $l$, as $h \rightarrow \infty$, one has

$$
\begin{aligned}
& J_{l}(h) \sim \sqrt{\frac{2}{\pi}} \frac{1}{\sqrt{h}} \cos \left(h-l \frac{\pi}{2}-\frac{\pi}{4}\right)+\mathrm{O}\left(h^{-\frac{3}{2}}\right), \\
& J_{l}^{\prime}(h) \sim-\sqrt{\frac{2}{\pi}} \frac{1}{\sqrt{h}} \sin \left(h-l \frac{\pi}{2}-\frac{\pi}{4}\right)+\mathrm{O}\left(h^{-\frac{3}{2}}\right),
\end{aligned}
$$

and hence

$$
G_{l}(h) \sim-\sqrt{\frac{2}{\pi}} \sqrt{h} \sin \left(h-l \frac{\pi}{2}-\frac{\pi}{4}\right)+\mathrm{O}\left(h^{-\frac{1}{2}}\right),
$$

which is independent of $\beta_{l}$.

We further recall that if $M(r)$ is the maximum of $F(z)$ on $|z|=r$, the order of the entire function $F$ is defined to be [21]

$$
\operatorname{ord}(F) \equiv \lim _{r \rightarrow \infty} \sup \frac{\log \log (M(r))}{\log (r)} .
$$

An example of an entire function of order $n$ is $e^{z^{n}}$. Our function $G_{l}$ in (1.6) can be also studied from the point of view of its order, as defined in (A6), and its relation with the genus [21].

Moreover, we can apply to Eq. (1.6) a theorem studied, among the others, by Watson [22], according to which, if $A$ and $B$ are real and $\nu>-1$, the function $A J_{\nu}+B z J_{\nu}^{\prime}$ has all its zeros real, except that it has 2 purely imaginary zeros when $\frac{A}{B}+\nu<0$. This implies that our $G_{l}(h)$ has only real roots if

$$
\beta_{l}+l \geq 0
$$

This condition is fulfilled by all forms (4.5)-(4.8) of $\beta_{l}$ considered in our paper.

The uniform asymptotic expansions of Bessel functions and their first derivative are due to Debye and Olver [6] and are used extensively in Ref. [3] and in our paper. They read as

$$
J_{l}(i h) \sim \frac{(i h)^{l}}{\sqrt{2 \pi}} \alpha_{l}^{-\frac{1}{2}} e^{\alpha_{l}} e^{-l \log \left(l+\alpha_{l}\right)} \Sigma_{1},
$$




$$
J_{l}^{\prime}(i h) \sim \frac{(i h)^{l-1}}{\sqrt{2 \pi}} \alpha_{l}^{\frac{1}{2}} e^{\alpha_{l}} e^{-l \log \left(l+\alpha_{l}\right)} \Sigma_{2}
$$

where [see also (2.13)-(2.19)],

$$
\begin{aligned}
& \Sigma_{1} \sim u_{0}+\frac{u_{1}}{l}+\frac{u_{2}}{l^{2}}+\frac{u_{3}}{l^{3}}+\cdots, \\
& \Sigma_{2} \sim v_{0}+\frac{v_{1}}{l}+\frac{v_{2}}{l^{2}}+\frac{v_{3}}{l^{3}}+\cdots,
\end{aligned}
$$

and, in particular,

$$
\begin{aligned}
u_{3}(\tau)= & \frac{1}{2} \tau^{2}\left(1-\tau^{2}\right) u_{2}^{\prime}(\tau) \\
& +\frac{1}{8} \int_{0}^{\tau}\left(1-5 \rho^{2}\right) u_{2}(\rho) d \rho \\
v_{3}(\tau)= & u_{3}(\tau)+\tau\left(\tau^{2}-1\right)\left[\frac{1}{2} u_{2}(\tau)+\tau u_{2}^{\prime}(\tau)\right] .
\end{aligned}
$$

[1] M. Schröder, Rep. Math. Phys. 27, 259 (1989).

[2] A. O. Barvinsky, A. Yu. Kamenshchik, and I. P. Karmazin, Ann. Phys. (N.Y.) 219, 201 (1992).

[3] I. G. Moss, Classical Quantum Gravity 6, 759 (1989).

[4] P. B. Gilkey, Invariance Theory, the Heat Equation and the Atiyah-Singer Index Theorem (CRC Press, Boca Raton, 1995).

[5] G. Esposito, Dirac Operators and Spectral Geometry (Cambridge University Press, Cambridge, England, 1998).

[6] F. W. J. Olver, Phil. Trans. R. Soc. A 247, 328 (1954).

[7] R. Wong, Asymptotic Approximations of Integrals (Academic, New York, 1989).

[8] G. H. Hardy, Divergent Series (Oxford University Press, Oxford, 1949).

[9] G. Esposito, Classical Quantum Gravity 16, 1113 (1999).

[10] G. Esposito, Classical Quantum Gravity 16, 3999 (1999).

[11] E. Elizalde, S. D. Odintsov, A. Romeo, A. A. Bytsenko, and S. Zerbini, Zeta Regularization Techniques with Applications (World Scientific, Singapore, 1994).

[12] K. Kirsten, Spectral Functions in Mathematics and Physics (Chapman \& Hall, London, 2001).
[13] E. Elizalde, Ten Physical Applications of Spectral Zeta Functions, Lecture Notes in Physics Vol. 855, 2nd ed. (Springer-Verlag, Berlin, 2012).

[14] M. Bordag, E. Elizalde, and K. Kirsten, J. Math. Phys. (N.Y.) 37, 895 (1996).

[15] M. Bordag, E. Elizalde, K. Kirsten, and S. Leseduarte, Phys. Rev. D 56, 4896 (1997).

[16] E. Elizalde, M. Bordag, and K. Kirsten, J. Phys. A 31, 1743 (1998).

[17] P. D. D'Eath and G. Esposito, Phys. Rev. D 43, 3234 (1991).

[18] I. G. Avramidi and G. Esposito, Commun. Math. Phys. 200, 495 (1999).

[19] E. Witten, arXiv:1805.11559.

[20] L. V. Ahlfors, Complex Analysis (McGraw-Hill, New York, 1966).

[21] R. P. Boas, Entire Functions (Academic Press, New York, 1954).

[22] G. N. Watson, A Treatise on the Theory of Bessel Functions (Cambridge University Press, Cambridge, England, 1944). 